\title{
Probability of Coverage Based Analysis of Distributed Antenna System and its Implementation on LTE Based Real-Time-Testbed
}

\author{
Peethala, Sundar Daniel Victor; Zarifeh, Nidal; Kaiser, Thomas
}

This text is provided by DuEPublico, the central repository of the University Duisburg-Essen.

This version of the e-publication may differ from a potential published print or online version.

DOI: https://doi.org/10.1109/ICUMT.2017.8255196

URN: urn:nbn:de:hbz:464-20180308-075047-5

Link: http://duepublico.uni-duisburg-essen.de/servlets/DocumentServlet?id=45571

Legal notice:

(C) 2017 IEEE. Personal use of this material is permitted. Permission from IEEE must be obtained for all other uses, in any current or future media, including reprinting/republishing this material for advertising or promotional purposes, creating new collective works, for resale or redistribution to servers or lists, or reuse of any copyrighted component of this work in other works.

Source: This is the author's version of an article that has been published in "2017 9th International Congress on Ultra Modern Telecommunications and Control Systems and Workshops (ICUMT)". Changes were made to this version by the publisher prior to publication. The final version of record is available at https://doi.org/10.1109/ ICUMT.2017.8255196 


\title{
Probability of Coverage Based Analysis of Distributed Antenna System and its Implementation on LTE Based Real-Time-Testbed
}

\author{
Daniel Peethala, Nidal Zarifeh, and Thomas Kaiser \\ Institut für Digitale Signalverarbeitung \\ Universität Duisburg-Essen \\ 47057 Duisburg, Germany \\ Email: \{daniel.peethala, nidal.zarifeh and thomas.kaiser\}@uni-due.de
}

\begin{abstract}
Distributed Antenna Systems (DASs) provide solutions to the future wireless challenges targeted for $5 \mathrm{G}$ systems using distributed remote radio heads (RRHs). In the case of a multi-user DAS, the sum rate can be increased by pairing the UEs with specific RRHs, thereby facilitating independent transmissions from different RRHs. In this work, probability of coverage metric is derived and used for the analysis of different DAS scenarios using selection transmission (ST) based pairing. This work also describes PHY level modelling of processing blocks, implementation details, hardware and procedures for DAS based on 3GPP LTE. The implementation details of realtime LTE based DAS testbed besides the relevant LTE procedures for the deployment of DAS are presented. Verification of the LTE based DAS testbed is achieved using the vector signal analyzer (VSA) software running on Keysight's mixed signal analyzer (MXA) and commercial LTE UEs. The results of our analysis based on probability of coverage metric in different DAS scenarios, i.e., with varied number of remote radio heads (RRHs) and targeted SINR at different UE positions are presented.
\end{abstract}

Index Terms - remote radio head (RRH), distributed antenna system (DAS), fifth generation mobile communication technology (5G), physical layer (PHY), medium access control layer (MAC), long term evolution (LTE), digital signal processor (DSP)

\section{INTRODUCTION}

In the recent years, distributed antenna system (DAS) has gained interest due to its viable solutions for cell coverage extension, high frequency re-use and improved latency [1], [2]. Multiuser DAS is one of the most promising techniques for $5 \mathrm{G}$ systems, especially for indoor [3] or outdoor hotspot coverage [4]. DAS mainly differs from a conventional collocated antenna system by employing geographical distribution of remote radio heads (RRHs). The RRHs are connected to a centralized processing unit using dedicated cables, usually optical fibers. DAS has proven to offer viable solutions to the challenges such as throughput improvement, call blocking rate

This work was conducted within the framework of the 5G-PPP COHERENT project, which is partially funded by the Commission of the European Union (Grant Agreement No.671639). reduction, coverage improvement and reduction in transmit power [5], [6].

Significant work [7]-[10] on DAS to understand the advantages and possible deployment strategies of DAS in comparison with collocated antenna systems has been done. With respect to the understanding of adaptations required in 3GPP LTE Rel. 9 standard to incorporate DAS functionality, not much work [11], [13] has been reported. As LTE based systems and networks are currently deployed in most of the countries, the benefits of DAS such as: significant reduction in number of handovers, coverage extension and improved throughput can be effectuated by some modifications in LTE processing blocks and procedures at the base station or EUTRAN Node B (eNodeB) in LTE and at the UE. The work proposes a set of exploitation methods and modifications of LTE PHY processing blocks for supporting the functionalities of DAS in an LTE system.

Selection transmission (ST) [5], a simple DAS technique, which is based on selecting a single RRH out of all the RRHs for transmission has gained considerable interest in DAS because it reduces the other cell interference (OCI) and retains the benefits of spatial diversity. In [12], a study on different schemes for selection transmission to increase the sum rate has been done in the case of a single cell multi-user downlink (DL) distributed antenna systems. However, in order to improve the sum rate of the cell, ST based pairing scheme, which pairs the UEs to the RRHs based on their distances or path losses is considered for our work. The ST based pairing offers opportunities to explore the re-use of time-frequency resources in the same cell. Probability of coverage metric is introduced to analyze different DAS scenarios. This metric provides reliable means to exploit the opportunities for pairing depending on the expected SINR at different UE positions. In the view of deploying this DAS technique in a standard 3GPP LTE Rel. 9 compliant system, this work also summarizes the study conducted by us so far with regard to the relevant LTE procedures like cell search, cell selection etc. as described in LTE standard [14]-[16]. 
The rest of the paper is organized as follows: in Section II, Pairing in DAS, where the system model is described, along with a description of probability of coverage metric, which is used for the analysis of different DAS scenarios. Section III describes the LTE procedures for the deployment of DAS and implementation aspects of DAS based real-time LTE testbed, which is implemented on texas instrument's (TI) TMS320C6670 DSP and ARM boards. In Section IV the simulation results for the analysis of DAS based on probability of coverage metric for different DAS scenarios at different UE positions for targeted throughput are presented. Section V concludes the paper.

\section{PAIRING IN DAS}

Fig.1 shows a typical DAS system, where the DAS eNodeB does all the processing of higher layers until physical layer and sends the time domain baseband signals to the RRHs via optical cables making use of baseband transmission standards like common public radio interface (CPRI) or open base station architecture initiative (OBSAI). Fig.1 also shows the general idea of having paired selection transmission technique where the RRH serves the nearest UEs. The simplest technique of ST based pairing as described in [12] is to pair UEs with RRHs based on the distances or path losses. In order to increase the sum rate using ST, a set of RRHs can use the same time, frequency and space resources thereby causing tolerable intra-cell interference. In some real-life scenarios, where the UEs are mostly stationary or less mobile and the need for sum rate is very high, for e.g. stadiums, theaters, shopping malls etc., one can pair the UEs with the RRHs so that the same scheduling resources of the pair can be reused in an other pair quite far, i.e., where the signal to interference plus noise ratio (SINR) is high enough to decode the channels.

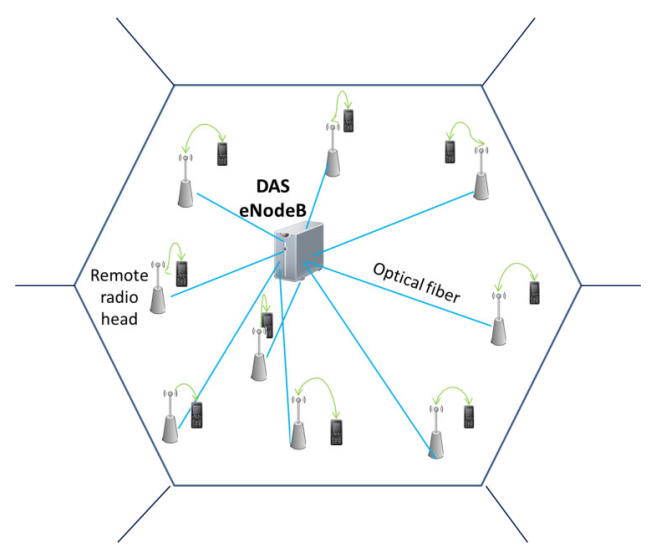

Fig. 1: DAS EnodeB with centralized processing

\section{A. System model}

We consider a cell, which consists of $N$ RRHs and $K$ UEs distributed over the cell. Each RRH can have multiple antennas as defined in the LTE standard, but here we just consider single-input single-output (SISO). The received signal at any $i^{t h}$ UE in the cell from $j^{\text {th }}$ RRH can be expressed as

$$
y_{i j}=h_{i, j} x_{j}+z_{i}
$$

where $h_{i, j}$ denotes the coefficients of small-scale fading. Rayleigh fading channel is considered here. $x_{j}$ represents transmitted signal from the $j^{\text {th }} \mathrm{RRH}$ port and $z_{i}$ represents the noise at the receiver. The probability distribution function of the received power, $r_{i j}$, based only on simple Rayleigh fading channel at the UE $i$ is given by:

$$
f_{r}\left(r_{i j}\right)=\frac{1}{2 \gamma^{2}} \exp \left(\frac{-r_{i j}}{2 \gamma^{2}}\right)
$$

where $2 \gamma^{2}=\frac{P_{j}}{d_{i, j}^{\alpha}}$ represents the mean received power of the signal, where we assume that transmitted power falls off based on $d_{i, j}$, i.e., the distance between the RRH port $j$ and the $i^{t h}$ UE and path loss exponent $\alpha . E\left(x_{j} x_{j}^{*}\right)=P_{j}$ represents the transmitted power of $j^{\text {th }} \mathrm{RRH}$.

\section{B. Probability of coverage}

In this subsection, we introduce the probability of coverage metric [19] for analyzing different selection based pairing. In an interference and noise limited system, i.e., when all the RRHs are transmitting, the received signal at the $i^{\text {th }} \mathrm{UE}$ is

$$
y_{i}=h_{i, i} x_{i}+\sum_{j=1, j \neq i}^{N} h_{i, j} x_{j}+z_{i}
$$

Signal to interference plus noise ratio (SINR) at $i^{t h}$ UE is given by

$$
S I N R_{i, j}=\frac{r_{i j}}{I+\eta}
$$

where $\eta$ is the noise power at the receiver and $I$ is the interference observed at $i^{t h} \mathrm{UE}$ is $\sum_{j=1, j \neq i}^{N} r_{i j}$. Probability of coverage based on SINR is given by

$$
\begin{array}{r}
p_{c}=P\left(S I N R_{i, j}>\Theta\right)=P\left(\frac{r_{i j}}{I+\eta}>\Theta\right) \\
=P\left(r_{i j}>\Theta(I+\eta)\right) \\
=E_{I}\left(\int_{\Theta(I+\eta)}^{\infty} \frac{d_{i, i}^{\alpha}}{P_{i, i}} \exp \left(\frac{-r_{i, i} d_{i, i}^{\alpha}}{P_{i, i}}\right) d r_{i, i}\right)
\end{array}
$$

where $\Theta$ is the minimum SINR expected at the UE to achieve the targeted throughput. $E_{I}$ represents the expectation over the interference at $i^{t h}$ UE. As the interference is independent to the received power and the interferers are independent as well, 5 can be derived as

$$
\begin{array}{r}
p_{c}=\int_{0}^{\infty} \ldots \int_{0}^{\infty} \exp \left(\frac{-\Theta\left(\sum_{j=1, j \neq i}^{K} r_{i, j}+\eta\right)}{P_{i, i}}\right) \\
\prod_{j=1, j \neq i}^{K} f_{r_{i, j}} d r_{i, j}
\end{array}
$$


After performing simple integrations, $p_{c}$ can be derived as

$$
p_{c}=\exp \left(\frac{-\Theta \eta d_{i, i}^{\alpha}}{P_{i, i}}\right) \prod_{j=1, j \neq i}^{K} \frac{1}{1+\Theta \frac{P_{i, j}}{P_{i, i}} \frac{d_{i, i}^{\alpha}}{d_{i, j}^{\alpha}}}
$$

\section{LTE PROCEDURES AND IMPLEMENTATION OF LTE BASED DAS TESTBED}

Firstly, we will summarize here our investigations about deployment of DAS in a LTE System, in the view of LTE procedures such as: cell search, cell selection, random access and multi-user scheduling of resources. Received signal strength indicator (RSSI), is defined in the standard [17] as the total signal power of each resource element (RE) including interference and noise. RE, which is 1 subcarrier times 1 symbol, is the smallest discrete part of the LTE frame, defined as $15 \mathrm{KHz}$ in frequency. In a typical LTE system, each cell is uniquely identified based on physical layer cell identity (cellID)number, that is broadcasted using primary synchronization sequence (PSS) and secondary synchronization sequence (SSS) channels. After turning on, the UE sets the radio frequency (RF) carrier frequency to the different center frequencies of the operator allowed bands according to E-UTRA absolute radio frequency channel number (EARFCN) defined in [18]. The UE then tries to find the PSS, which is a part of the synchronization signals transmitted by the eNodeB. ZadoffChu sequences, which have good auto-correlation properties in frequency domain, are used to generate the PSS. After detection of the PSS signals, UE measures the RSSI of that cell. After completion of the cell search, the cell with the greatest RSSI value will be selected by the UE and decides to camp on the cell, which is defined in LTE as cell selection procedure [14].

In DAS, we assume different CellIDs can be transmitted from different RRHs, so that the cell selection procedure in a typical LTE cell can be applied for RRH selection. The pairing can be achieved w.r.t. the RSSI values, which is a good metric of the observed SINR at the UE location w.r.t. different RRHs. After the UE gets attached to a RRH, the eNodeB can analyze and exploit the opportunities to reuse the resource blocks (RBs) to different UEs based on CQI and the observed SINR.

Secondly, regarding our DAS implementation on Real-time LTE testbed, our previous work [13] explains the details of the processing blocks required for implementing the DAS functionality using LTE downlink shared channel (DLSCH). Fig.2 describes the LTE downlink shared channel (DLSCH) processing blocks at the transmitter along with the introduction of DAS Engine. DAS Engine processing block mainly multiplexes the IQ time domain data of different UEs with different RRHs, in accordance to the ST based pairing. Besides multiplexing, DAS Engine provides varied opportunities for network MIMO, coordinated Multi-Point transmission (CoMP), Multi-user MIMO etc. for DAS.

Fig.3 describes, the software partitioning of the PHY layer on the DSP, besides showing the hardware blocks: Antenna interface module (AIF2) for baseband IQ signals transmission to the different RRHs, ARM where MAC and protocol stack

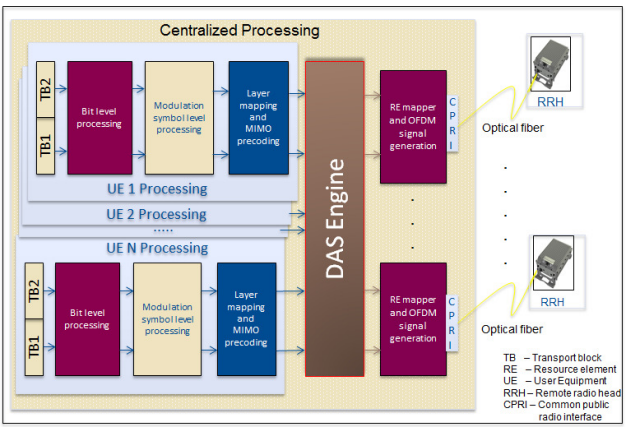

Fig. 2: DAS Processing blocks for DLSCH

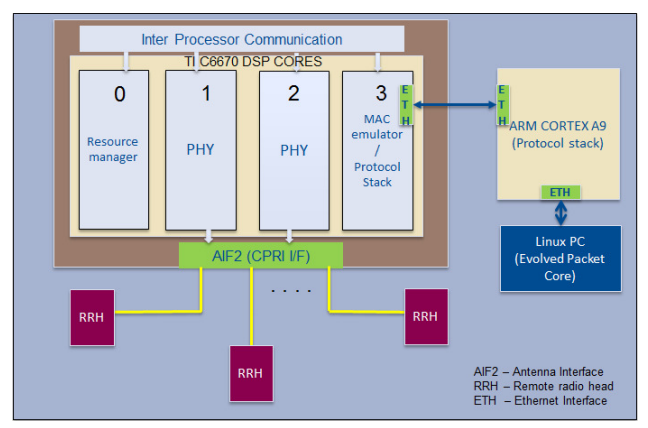

Fig. 3: Hardware Implementation blocks

(PS) runs and the linux PC where evolved packet core (EPC) runs. The DSP communicates with protocol stack via Gigabit Ethernet. Protocol stack communicates with evolved packet core (EPC), running on a linux PC via Gigabit Ethernet. Although DSP is capable of processing for many users, without loss of generality, as a first step we have selected a use case with only 2 UEs per subframe and 2 RRHs. The current LTE testbed is fully functional to attach many commercial UEs and to communicate with them.

\section{RESULTS}

In Section II, the probability of coverage metric is derived, which is verified with an empirical model using Rayleigh distribution and Monte-Carlo simulations in MATLAB and the results match exactly. A single cell of 20 meters radius is considered for analysis. Within the cell, different RRH distributions i.e., the number of RRHs per cell is considered. The number of RRHs per cell has been selected to be 7 , 19, 37, 61 and 91 based on the optimum circular packing of RRHs in the hexagonal area. Different UE positions are considered in the same RRH coverage area, i.e., $R R H_{0}$ or the center of the cell. The UE positions are described by the radial distance factor i.e., ratio of the UE radial distance to the RRH coverage radius. Fig.4 and Fig.5 represent the layout of the cell and number of RRHs packed per cell 7, 19, 37 and 61. Different UE positions as represented with ' $\mathrm{x}$ ' and a downpointed triangle represents the RRHs, numbered starting with 0 .

In Fig.6, the probability of coverage for a targeted SINR of $15 \mathrm{~dB}$ is simulated for different DAS scenarios. In this 


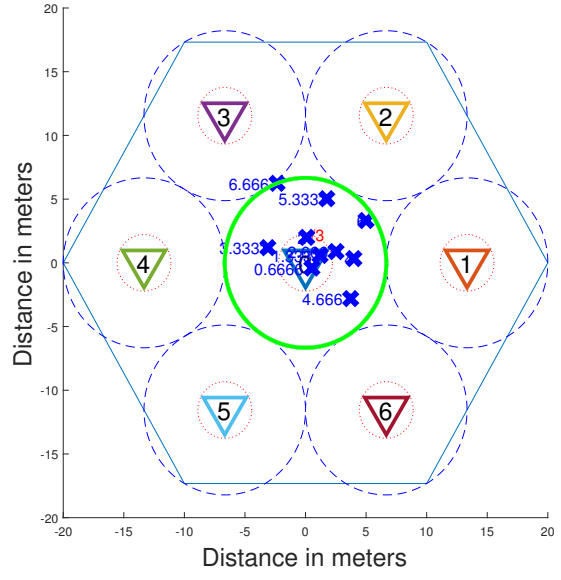

(a)

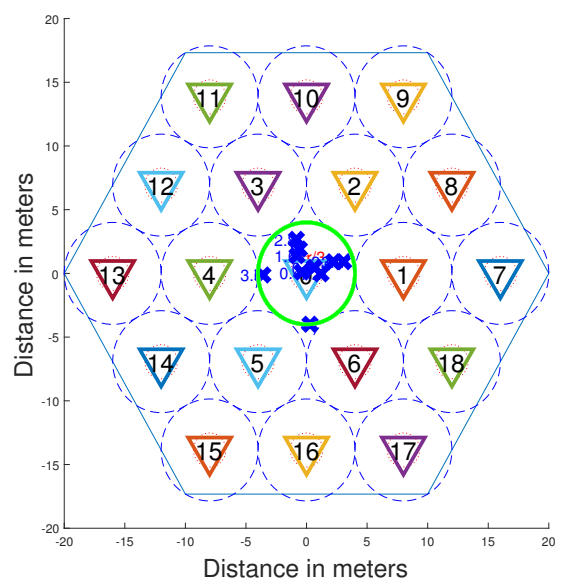

(b)

Fig. 4: Different packing of RRHs in a cell, (a) 7 and (b) 19 RRHs

scenario, the number of RRHs per cell is varied to observe it's affect on the probability of coverage for a targeted SINR. The curves show that there is a slight degradation in the probability of coverage if the number of RRHs in the same area is increased, but the more the number of RRHs the more probable will be the reutilization of time-frequency resources in the same area. Therefore, the advantage of having more RRHs outweighs the slight degradation. Fig.7 and Fig.8 shows the probability of coverage w.r.t. different expected SINR, i.e., $0,5,10,20$ and $25 \mathrm{~dB}$, in case of $7 \mathrm{RRHs}$ and $19 \mathrm{RRHs}$ respectively. From these curves, depending on the targeted SINR, i.e., the targeted throughput the probability of coverage decreases rapidly if the UE is distant than $1 / 3$ of the RRH radius from the location of RRH. Nevertheless, compared to the multiple access in LTE standard, where non-overlapping scheduling of resources is defined, one can make use of the probability of coverage at the UE positions and throughput requirements of the UE for exploiting the opportunities of

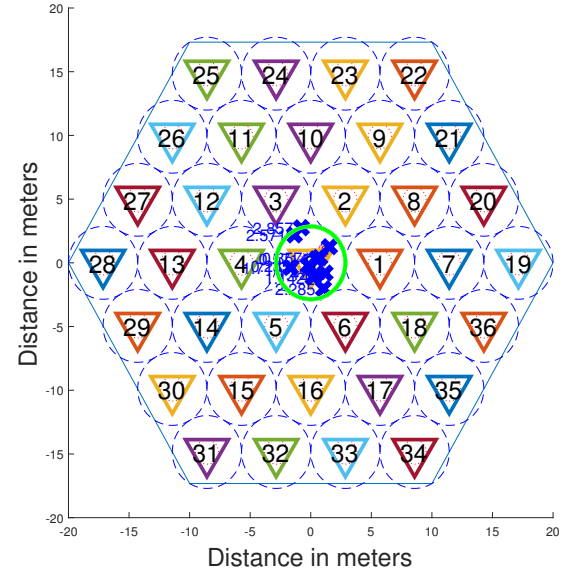

(a)

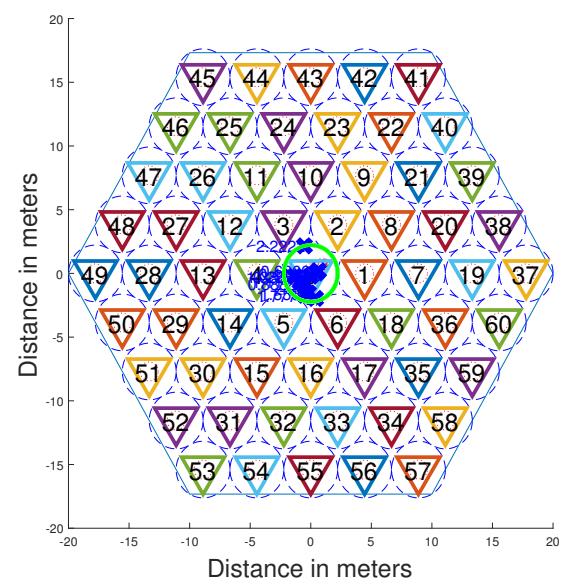

(b)

Fig. 5: Different packing of RRHs in a cell, (a) 37 and (b) 61 RRHs

scheduling overlapping resources.

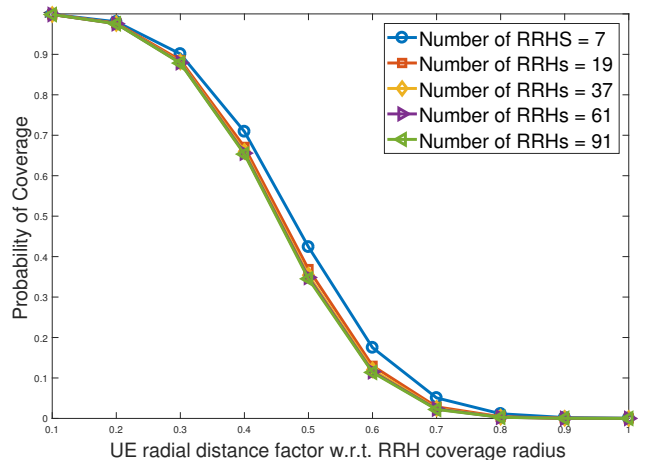

Fig. 6: Probability of Coverage for a targeted SINR of $15 \mathrm{~dB}$ in varied UE positions and the number of RRHs

Regd. the trials with real-time LTE based DAS testbed, we have made use of Keysight's MXA and VSA signal mea- 


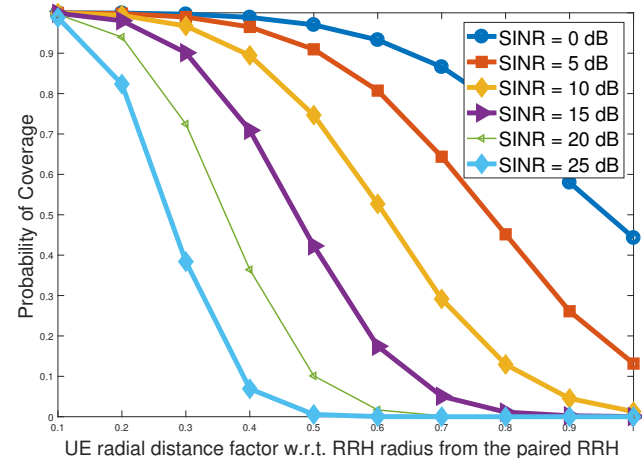

Fig. 7: Probability of Coverage for different targeted SINR in a cell with 7 RRHs in varied UE positions

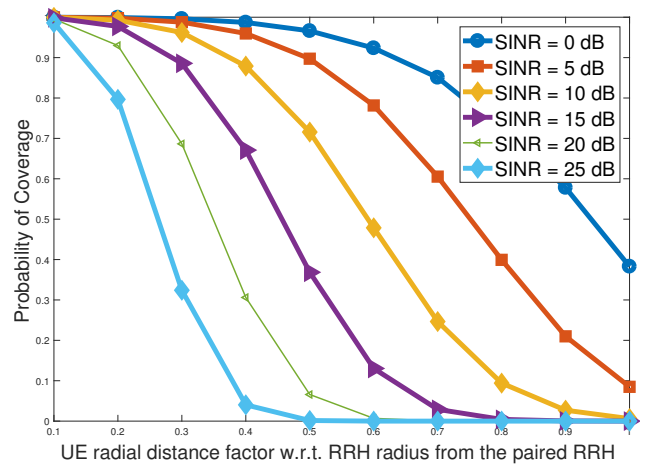

Fig. 8: Probability of Coverage for different targeted SINR in a cell with 19 RRHs in varied UE positions

surement setup for LTE PHY layer analysis, which provides in-depth analysis of the decoded LTE channels, error vector magnitude (EVM). The current implementation is a simple DAS technique i.e., pairing based on distance of the UE. In this approach the nearest user to the RRH would be served by that RRH. To validate this approach we have implemented LTE eNodeB with DAS functionality as described in Section III. To validate the pairing, overlapping RB allocation / scheduling for UEs was implemented, in contrast to standard LTE. For the measurement, LTE single stream (SISO) transmission is configured for both the UEs. Different Radio network temporary identifiers (RNTIs) are configured for the UEs. Fig.9 shows the testbed which is a microTCA chassis that contains a TI DSP board and RRH board apart from the microTCA controller. The DSP and RRH are connected via CPRI using optical cable. Keysight's VSA software running on MXA signal analyzer is capable of real-time capturing and decoding of time domain LTE signals over the air. Fig.10 shows the decoded IQ constellation along with the allocated RBs provided by the VSA software. The measurement setup can decode the DLSCH channels transmitted through the implemented DAS Engine, which verifies the real-time LTE based DAS testbed implementation.

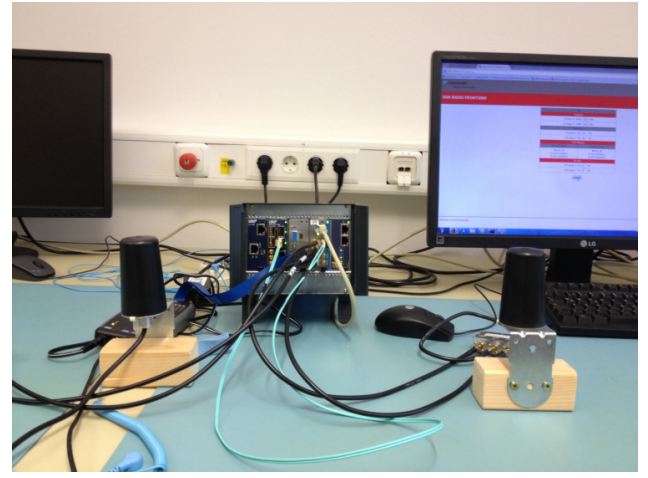

Fig. 9: LTE testbed

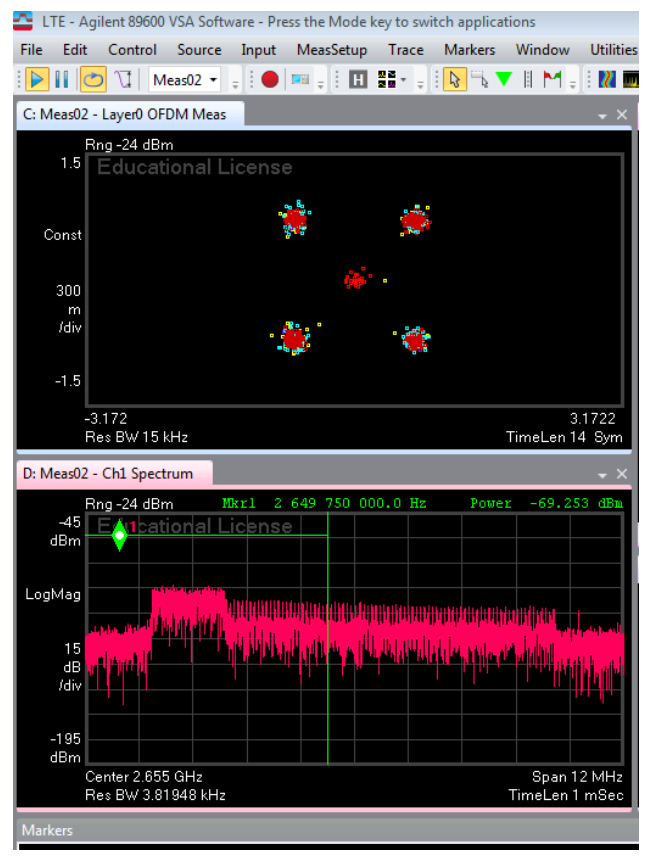

Fig. 10: Measurement results

\section{CONCLUSION}

In this paper, we have described a DAS system model and derived the probability of coverage metric for analyzing different DAS scenarios i.e., varied number of RRHs in a cell and targeted SINR at different UE positions. In the view of deployment of DAS in the standard 3GPP LTE Rel. 9 system, investigations related to initial access procedures of UE such as: RSSI measurements, Cell search and Cell selection, besides scheduling of resources at eNodeB are presented. The implementation details of DAS on a realtime LTE system and proposed required adaptations to LTE DLSCH processing blocks for DAS functionality, i.e., the DAS Engine is described. Different RRH packing i.e with 7, 19, 37 and 61 RRHs per cell scenarios are presented. The probability of coverage ensures that the required throughput at the UE is achieved statistically, based on the channel model. The probability of coverage at different UE positions based on targeted SINR, which is directly related to the required 
throughput at the UE position, in different DAS scenarios is analyzed. From the presented results we conclude that, depending on the targeted SINR, i.e., the targeted throughput the probability of coverage decreases rapidly at the UE positions greater than $1 / 3$ of the RRH coverage radius. However, compared to the traditional multiple access in LTE standard, where non-overlapping scheduling of resources is defined, one can make use of the probability of coverage at the UE position, throughput requirement of the UE in order to exploit the opportunities to schedule overlapping resources. Hence pairing RRHs with UEs enables to find the opportunities within the cell to re-use the RBs, which can significantly improve the spectral efficiency. Therefore DAS provides viable solutions for the future $5 \mathrm{G}$ mobile communication systems. The future work is oriented towards finding algorithms for scheduling the overlapping RBs at different RRHs based on the probability of coverage at UEs for the targeted throughput, besides considering other performance indicators to make the system more realistic.

\section{REFERENCES}

[1] R. Heath, S. Peters, Y. Wang, and J. Zhang, "A current perspective on distributed antenna systems for the downlink of cellular systems," IEEE Commun. Mag., vol.51, no.4, pp.161-167, April 2013.

[2] L. Dai, S. Zhou, and Y. Yao, "Capacity analysis in CDMA distributed antenna systems," IEEE Trans. Wireless Commun., vol.4, no.6, pp.26132620, Nov. 2005

[3] H. Osman, H. Zhu, D. Toumpakaris, and J. Wang, "Achievable rate evaluation of in-building distributed antenna systems," IEEE Trans. on Wireless Communications vol. 12, no. 7, pp. 35103521, Nov. 2013

[4] X.-H. You, D.-M. Wang, B. Sheng, X.-Q. Gao, X.-S. Zhao, and M. Chen, Cooperative distributed antenna systems for mobile communications, IEEE Wireless Communications pp. 3543, Jun. 2010.

[5] W. Choi, and J. G. Andrews, "Downlink performance and capacity of distributed antenna systems in a multicell environment," IEEE Trans. Wireless Commun., vol.6, no.1, pp.69-73, Jan. 2007.

[6] R. Bosisio, and U. Spagnolini, "Interference Coordination Vs. Interference Randomization in Multicell 3GPP LTE System," IEEE Wireless Communications and Networking Conference, 2008. WCNC 2008., vol., no., pp.824-829, March 31 2008-April 32008.

[7] Z. Liu, and L. Dai, "A Comparative Study of Downlink MIMO Cellular Networks With Co-Located and Distributed Base-Station Antennas," IEEE Trans. Wireless Commun., vol.13, no.11, pp.6259-6274, Nov. 2014.

[8] K. T. Truong, and R. W. Heath, "The viability of distributed antennas for massive MIMO systems," Asilomar Conference on Signals, Systems and Computers, 2013, vol., no., pp.1318-1323, 3-6 Nov. 2013.

[9] H. Q. Ngo, A. Ashikhmin, H. Yang, E. G. Larsson, and T. L. Marzetta, "Cell-Free Massive MIMO: Uniformly great service for everyone," IEEE 16th International Workshop on Signal Processing Advances in Wireless Communications (SPAWC), 2015, vol., no., pp.201-205, June 28 2015July 12015.

[10] A. A. M. Saleh, A. J. Rustako, and R. Roman, "Distributed Antennas for Indoor Radio Communications," IEEE Trans. Commun.", vol.35, no.12, pp.1245-1251, December 1987.

[11] Y. D. Beyene, R. Jantti, and K. Ruttik, "Cloud-RAN Architecture for Indoor DAS," IEEE Access, vol.2, no., pp.1205-1212, 2014.

[12] K. Heejin, S. Lee, and I. Lee, "Sum Rate based Transmission Selection Schemes in Distributed Antenna Systems," Vehicular Technology Conference (VTC Spring), 2013 IEEE 77th, Dresden, 2013 pp. 1-5.

[13] S. D. V. Peethala, T. Kreul and T. Kaiser, "Real-time Testbed for Validating Distributed Antenna Scenarios," WSA 2016; 20th International ITG Workshop on Smart Antennas, Munich, Germany, 2016 pp. 1-4.

[14] 3GPP TS 36.213 V9.2.0, Physical layer procedures (Release 9).

[15] 3GPP TS 36.212 V9.2.0, Multiplexing and channel Coding (Release 9).

[16] 3GPP TS 36.211 V9.1.0, Physical Channels and Modulation (Release 9).

[17] 3GPP TS 36.214 V9.1.0, Physical layer - Measurements (Release 9).
[18] 3GPP TS 36.101 V9.3.0, User Equipment (UE) radio transmission and reception (Release 9).

[19] K. Govindan, K. Zeng and P. Mohapatra, "Probability Density of the Received Power in Mobile Networks," IEEE Transactions on Wireless Communications, vol. 10, no. 11, pp. 3613-3619, November 2011. 